\title{
Active and passive thermography for defect detection in polymer joints
}

\author{
by H. Leicht*, M. Heilig*, C. Pommer*, E. Kraus*, B. Baudrit* \\ *SKZ - German Plastics Center, SKZ - KFE gGmbH, Joining and Surface Engineering, \\ Friedrich-Bergius-Ring 22, 97076 Wuerzburg, Germany, h.leicht@skz.de
}

\begin{abstract}
Non-destructive testing (NDT) is a growing field in quality monitoring in the plastics industry, as it offers the possibility to lower the expenses for quality assurance without lowering the quality level. Passive thermography is being established as a method for automatic on-line monitoring of the weld quality in joined plastics. Active thermography is an established method in quality assurance of composite materials, but further experience is necessary in the field of polymer joints. In this work, passive and active thermography have been evaluated for their sensitivity to common defects known in weld joints and adhesive bonds of polymers.
\end{abstract}

\section{Introduction}

Joining of plastics is usually one of the last steps in the value chain and flaws inside of joints quickly lead to a severe cut in the durability of the product. Today the most common way of testing polymer joints are destructive tests [1, 2]. But these lead to the problem that only few (representative?) parts may be inspected. Also the economic drawbacks of destroying finished parts are clearly visible. Therefore it is highly desirable to have an NDT method which constantly evaluates the joint integrity on-line or at-line [3]. At this point, thermographic measurements have a high potential to fulfil this need in an elegant way, as they are quick, easy to use (non-contact) and cheap compared to other NDT methods. Moreover polymers are highly suitable for quantitative thermographic measurements, as most common polymers have high emission coefficients of around 0.95 [4]. This leads to quiet small errors because of a misfit in emission coefficient.

Generally, thermography can be divided into two different fields, passive and active thermography, depending on the origin of the observed heat dissipated in the material [5]. Passive thermography uses the inherent heat in the material which originates from the production process. Because of the high temperature rise in welding processes, this method is highly suited for the on-line evaluation of welded joints.

In active thermography heat is dissipated actively in the material by the use of an excitation source, whereby one has to limit the dissipated energy to prevent damaging of the material. This method is suitable for the at-line examination or the examination of structures in use (lifetime prediction). It can be used for both adhesive bonds and welded joints, as no inherent heat is needed.

In our studies passive thermography has been used to detect typical process deviations, like surface defects or different humidities, during hot plate, infrared and ultrasonic welding of polymers.

Also active thermography, by means of lock-in thermography, has been used to detect common defects in hot plate welded and adhesive bonded polymer joints. The chosen defects are typical for industrial applications, e.g. air inclusions or contaminations of the joint area. Two different ways of excitation, optical and ultrasound, have been used.

\section{Theoretical background}

\subsection{Passive thermography}

In passive thermography the thermographic system is integrated directly into the welding process thus enabling an on-line evaluation of the process. It is possible to directly examine the thermographic data for the picture with the highest contrast, but this way of data examination is a quiet tedious approach. Another way of data analysis is the differential picture method that has been used in this work. The differential picture consists of a matrix of differential pixels. As shown in Eq. (1), the differential pixels $D P$ are calculated by the temperature difference between each pixel of the current measurement $C P$ and a reference picture. The reference picture is created by averaging a number $n$ of thermographic measurements $R P_{i}$ of a process with known quality, e.g. by means of tensile strength. An illustration of the method is shown in figure 1 .

$$
D P=C P-\frac{1}{n} \cdot \sum_{i=1}^{n} R P_{i}
$$




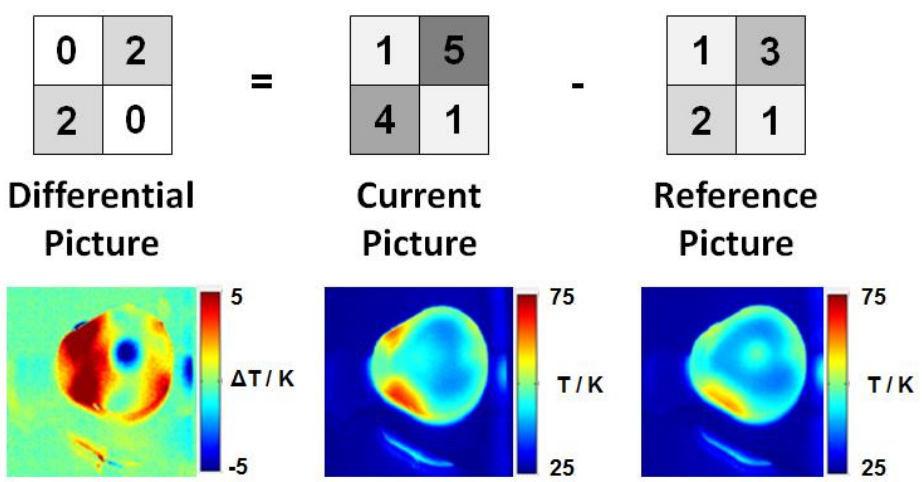

Fig. 1. Schematic of the differential picture method illustrated by pictures with four pixels and different grey values (1 to 5; top). Example for the measurement of an industrial part with a defect prior to welding (bottom).

The differential picture is highly sensitive to deviations in surface temperature thus providing an easy access to the detection of process interferences. Furthermore the histogram of the differential picture allows it to define process boundaries, thus enabling an automatic on-line quality evaluation.

\subsection{Active thermography}

Active thermography may be divided into different fields, depending on the waveform and the source of the excitation [6]. When using a periodic waveform of excitation, the method is referred to as lock-in thermography, whereas the use of a pulsed excitation would be called pulsed-phase thermography [7]. In lock-in thermography [8] the excited periodic signal on the parts surface is observed several times per modulation and usually gets fast Fourier transformed (FFT) into the frequency domain. Amplitude and phase pictures of the thermal structures are then generated by examining the generated data at the lock-in frequency $[9,10,11]$. The amplitude and phase pictures usually contain information on flaws and, depending on the kind of excitation, other thermal structures inside the part, as these lead to a shift in the amplitude and phase of the thermal waves. Especially the phase pictures gain a high interest during investigation as they have shown an enhanced depth range and are insensitive to several interferences [12]. As there are several ways of excitation the two relevant for this paper will be explained in the following.

In OLT (optical lock-in thermography) the heat is generated on the surface of the inspected part by absorption of the light emitted by optical sources, e.g. halogen lamps. By modulation of the brightness of the lamps a thermal wave is generated on the surface of the part, which propagates into the material, gets reflected by thermal boundaries on the inside and may be analysed by the lock-in method. The achievable depth range of the thermal waves is defined by the thermal diffusion length $\mu$, which may be calculated by Eq. (2) using the thermal diffusivity $\alpha$ of the material under investigation and the lock-in frequency $f[13,14]$.

$$
\mu=\sqrt{\frac{\alpha}{\pi \cdot f}}
$$

A high thermal diffusion length is needed to investigate structures in a greater depth. According to Eq. (2) this directly leads to the need of low lock-in frequencies and thus following long measurement times as several cycles of excitation have to be observed. In plastics the measurement time can reach several ten minutes in order to achieve a depth range of several millimetres, as their thermal diffusivity is low e.g. compared to metals. On the other hand it is possible to examine structures up to a few square metres in a single measurement [15].

During the measurement, every structure which affects the thermal transport, e.g. fibres in glass-fibre reinforced plastics (GFRPs) or flaws, is a thermal boundary to the thermal waves. That is why OLT provides an overview of all thermal structures regardless if they are defects or not $[16,17]$. So it is necessary to search for deviations of the known structures in order to recognize defects.

Contrary to the heat generation on the surface during OLT, in ULT (ultrasound lock-in thermography) the heat is generated inside the object under investigation $[18,19,20]$. Usually the dissipation of elastic waves near defect sites is way higher than in the rest of the material, which leads to an observable heat generation at these sites. The higher attenuation of elastic waves near defect sites emerges e.g. from local changes in material stiffness or a higher mechanical loss angle by interfacial friction [21, 22]. When the ultrasonic waves are amplitude modulated, the defect sites are initial points of thermal waves with the same modulation frequency as the ultrasonic waves, which may also be analysed by the lock-in method [23]. As the thermal waves emerge from the defect sites in ULT, the depth range is highly increased compared to the reflective measurements in OLT. Nevertheless the depth range is affected by parameters as input power, detector noise or thermal diffusion length [24]. During the examination of simple objects, e.g. tiles, the generation of standing waves is possible, which leads to an interfering pattern in the thermographic data. These patterns can effectively be suppressed by modulating the frequency of the ultrasonic source [14, 25]. 


\section{Materials and methods}

For the experiments a thermographic camera TIM160 (Micro-Epsilon Messtechnik GmbH \& Co. KG, Ortenburg, Germany) was used. The camera possesses an uncooled focal plane array detector with $160 \times 120$ pixels and a maximum readout frequency of $120 \mathrm{~Hz}$.

The used materials are listed in table 1. For adhesive bonding a two component polyurethane adhesive (Weiss Cosmo PU-200.330) has been used.

Table 1. List of polymers, their tradename and abbreviations used in this work.

\begin{tabular}{|l|l|l|}
\hline Polymer & Trade name & Abbreviation \\
\hline Polyamide 6.6 & Du Pont Zytel 101 NC010 & PA66 \\
\hline Polyamide 6.6 with 25 \% glass fibres & BASF Ultramid B3WG5 & PA6 GF25 \\
\hline Polyvinylidene fluoride & Agru PVDF 30.600.2010.06 & PVDF \\
\hline Polyvinyl chloride & BEN MEDUR PVC-U & PVC \\
\hline Glass fibre reinforced polyamide & Bond Laminates Tepex dynalite 102-RG600 & GFRP-PA \\
\hline Glass fibre reinforced polypropylene & Bond Laminates Tepex dynalite 104-RG600 & GFRP-PP \\
\hline
\end{tabular}

Hot plate welding and infrared welding have been performed using a welding machine K2150 (bielomatik Leuze $\mathrm{GmbH}+$ Co. KG, Neuffen, Germany) with the thermographic camera placed right above the centre of the weld area. For infrared welding the hot plate has been replaced by an short-wavelength infrared emitter. For ultrasonic welding a welding machine 2000 X (Branson Ultraschall $\mathrm{GmbH}$, Dietzenbach, Germany) has been used. The thermographic camera had direct view onto the weld area and the contact area to the sonotrode. If not stated otherwise, the polymers have been dried for $18 \mathrm{~h}$ at $75^{\circ} \mathrm{C}$ prior to welding.

The emissivities of the polymers have been determined in a temperature range between $105^{\circ} \mathrm{C}$ and $195^{\circ} \mathrm{C}$ using a reference adhesive tape with known emissivity of 0.95 . The measurements have been performed inside a blackbox to minimize the effect of reflections.

For evaluation of the data in passive thermography proprietary software was used in order to automatically generate the differential-pictures process-accompanying. To create the reference data needed for the calculation of the differential-pictures within the software, the mean-value of the thermographic data of at least five welds for each process was used to account for statistical influences.

Active thermography was performed using a self-built setup consisting of two halogen lamps controlled by a frequency generator 4165 (PeakTech Prüf- und Messtechnik GmbH, Ahrensburg, Germany) for OLT and a commercially available IrNDT ultrasonic excitation source (AT - Automation Technology GmbH, Bad Oldesloe, Germany) for ULT. The lowest achievable lock-in frequency (sinoidal) of both systems has been $1.0 \mu \mathrm{Hz}$. In addition to the amplitude modulation for lock-in measurements, the frequency of the ultrasonic source in ULT has been modulated between $19.1 \mathrm{kHz}$ and $20.9 \mathrm{kHz}$ using a modulation frequency of $20.0 \mathrm{~Hz}$. For data acquisition in both methods at least 10 periods have been recorded using an at least 80 times higher recording frequency than the lock-in frequency.

FFT of the generated data has been executed with proprietary software.

\section{Passive Thermography}

\subsection{Hot plate and infrared welding}

In order to test the defect detection capabilities of the developed system for passive thermography, hot plate welding has been performed with different polymers and common defects influencing the welding process. Figure 2 shows the differential pictures of three surface defects simulated with PA6 GF25, which might easily occur during transport and handling of plastic parts in industrial use.
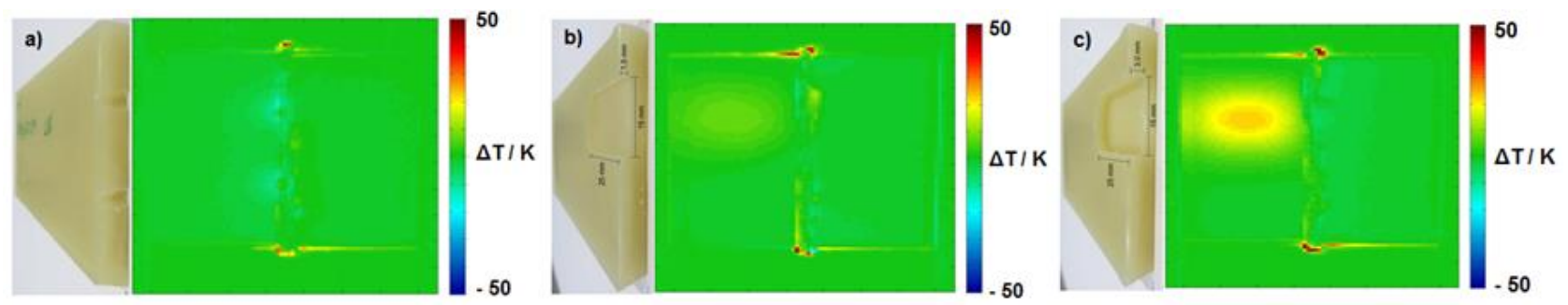

Fig. 2. Photographs prior to welding (left) and differential pictures (right) of hot plate welded PA6 GF25 sheets. The sheets have been prepared with two notches (a) as well as with wall thickness reductions of $1.5 \mathrm{~mm}$ (b) and $3.0 \mathrm{~mm}$ (c) within the weld area to simulate the effect of surface defects during welding.

The prepared notches can be clearly detected by passive thermography, as they create a cold zone in the weld area. The cold zones follow from a decreased heat transfer from the hot plate into the polymer as the polymer behind the 
notches is not in close contact with the hot plate. This supresses the conductive heat transfer, so the only way of heat transfer between hot plate and polymer is through radiation. With a growing time between welding and thermographic evaluation of the weld zone the surface temperatures tend to blur through temperature homogenization in the polymer part. Thus the moment of evaluation is of high importance for the detection of defects.

Furthermore two grooves with different depth on the backside of the PA6 GF25 sheets have been tested. In industrial use similar faults can arise e.g. through inhomogeneous wall thicknesses or missing structures on the inside. These defects can be detected by hot zones in the area with a smaller wall thickness, as the missing material leads to a higher temperature increase in the remaining polymer. A greater reduction in wall thickness also leads to a higher temperature increase, so the differential picture also contains information on the severity of the flaw.

The sensitivity of passive thermography to process deviations, e.g. divergent temperatures of the hot plate, is shown in figure 3
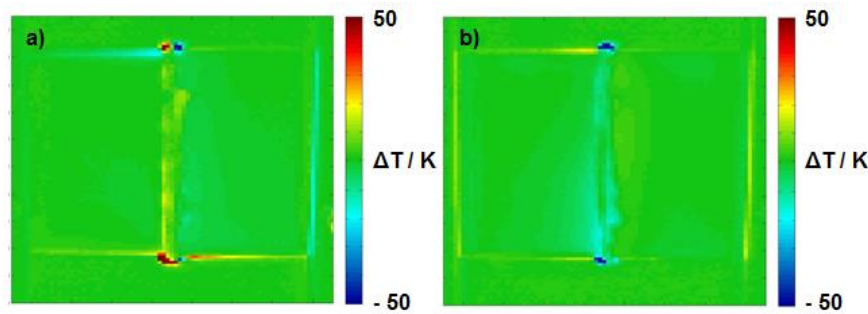

Fig. 3. Differential pictures of hot plate welded PA6 GF25 sheets.

The temperature of the hot plate has been raised (a) or lowered (b) by $10^{\circ} \mathrm{C}$ to simulate the effect of process deviations.

A higher temperature of the hot plate leads to a higher conductive heat transfer into the polymer, which can be seen in the differential pictures as a hot zone around the weld area. Contrary the smaller heat transfer due to a smaller temperature of the hot plate leads to a cold weld zone.

An overview over the detectability of the considered defects in comparison to mechanical tests (tensile test) and visual inspection after welding is shown in table 2.

Table 2. Detectability of different defects in hot plate welding by passive thermography, mechanical test (e.g. tensile test) and visual inspection after welding.

\begin{tabular}{|c|c|c|c|}
\hline \multirow{2}{*}{ Kind of defect } & \multicolumn{3}{|c|}{ Detectable by } \\
\hline & Passive thermography & Mechanical tests & Visual inspection \\
\hline \multicolumn{4}{|c|}{ Process deviations } \\
\hline $\begin{array}{l}\text { Hot plate temperature } \\
\text { (up to } \pm 5 \% \text { ) }\end{array}$ & Yes & No & No \\
\hline $\begin{array}{l}\text { Heating time } \\
\text { (up to } \pm 10 \% \text { ) }\end{array}$ & Partly & No & No \\
\hline \multicolumn{4}{|c|}{ Part deviations } \\
\hline Surface roughness & Yes & Yes & Partly \\
\hline Surface defects & Yes & Yes & Yes \\
\hline $\begin{array}{l}\text { Changes in } \\
\text { Wall thickness } \\
(1.5 \mathrm{~mm} \text { up to } 3.0 \mathrm{~mm})\end{array}$ & Yes & No & No \\
\hline $\begin{array}{l}\text { Polymer humidity } \\
\text { (up to } 1.23 \% \text { ) }\end{array}$ & No & No & No \\
\hline
\end{tabular}

As shown previously, process deviations like a change in hot plate temperature or heating time are detectable by passive thermography, yet the detectability of changes in heating time are dependent on the used polymer. The process deviations in the considered range (hot plate temperature $\pm 10{ }^{\circ} \mathrm{C}$, heating time $\pm 10 \%$ ) have not been detectable by mechanical tests and visual inspection. The same applies to changes in wall thickness. Surface defects could be detected by all used methods; especially the effect on mechanical testing is showing crucial impact of these defects on tensile strength and thus quality of the weld. Similar observations have been made for changes in surface roughness, but with one minor difference. During visual inspection they can only be seen for a roughness around $0.6 \mathrm{~mm}$. Changes in polymer humidity (up to $1.23 \%$ ) prior to hot plate welding have neither been detectable by passive thermography nor by the other methods. 
The detectability of surface defects by passive thermography during infrared welding is shown in figure 4 using PVDF as an example. Surface contaminations which change the absorption properties of the polymer surface are easily detectable in differential pictures. The ink used to blacken half of the weld surface leads to a high absorption of the infrared radiation and thus to a higher temperature rise compared to a non-blackened surface. Furthermore the higher absorption on the polymer surface leads to a lower temperature in the regions behind the weld zone, detectable as cold areas.

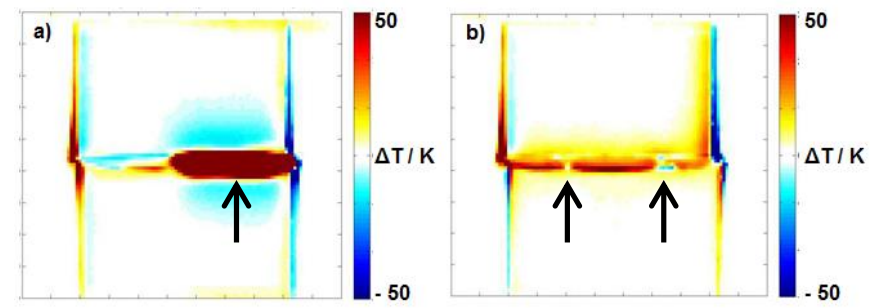

Fig. 4. Differential pictures of infrared welded PVDF sheets.

Half of the welded area has been blackened with ink (arrows; a) in order gain a higher absorption of the infrared radiation on the surface of the weld area.

Additionally sheets with two notches (indicated by arrows) in the weld area have been prepared to simulate the effect of surface defects (b).

Surface defects like notches lead to a local reduction in surface temperature. Contrary to the observation in hot plate welding these cold spots will not result from a different heat transfer but rather from geometrical effects, as the weld bead is observably smaller in the region of the notches. Additionally an increased surface roughness of around $0.2 \mathrm{~mm}$ can be detected due to a temperature decrease in the weld area.

An overview over the detectability of the considered defects in comparison to mechanical tests (tensile test) and visual inspection after welding is shown in table 3.

Table 3. Detectability of different defects in infrared welding by passive thermography, mechanical test (e.g. tensile test) and visual inspection after welding.

\begin{tabular}{|l|c|c|c|}
\hline \multirow{2}{*}{ Kind of defect } & \multicolumn{2}{|c|}{ Detectable by } & Visual inspection \\
\cline { 2 - 4 } & Passive thermography & Mechanical tests & No \\
\hline Surface roughness & Yes & Yes & Yes \\
\hline Surface defects & Yes & Yes & Yes \\
\hline Blackened surface & Yes & Yes \\
\hline
\end{tabular}

Changes in surface roughness have been detectable by passive thermography and mechanical tests. Same applies for surface defects and changes in the absorption properties, e.g. by a blackened surface, whereas they have also been detectable by visual inspection. Thus in infrared welding all considered defects, which had an adverse effect on mechanical strength of the weld could be detected by passive thermography.

\subsection{Ultrasonic welding}

The influence of the polymer humidity, which might occur through changing storage conditions insufficient drying of hygroscopic polymers in industrial use, is shown in figure 5.
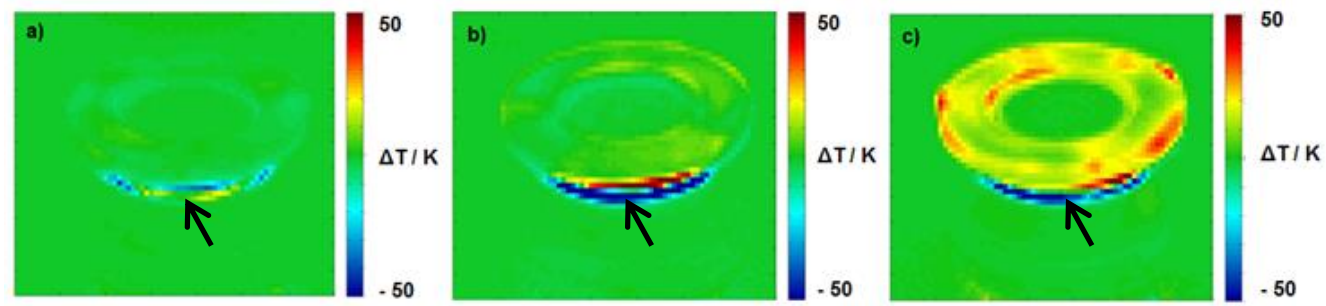

Fig. 5. Differential pictures of ultrasonic welded PA6 GF25 DVS specimens.

The humidity of the specimens prior to welding has been $0.32 \%$ (a), $0.45 \%$ (b) and $3.84 \%$ (c).

The weld area is indicated by an arrow. 
A growing water content in the polymer leads to a higher attenuation of the ultrasonic waves in the bulk polymer. Consequential the temperature in the weld zone is drastically getting smaller whereas the temperature of the contact area to the sonotrode is rising, as more energy gets converted into heat on the way to the weld zone. This leads to the production of parts with a low weld quality as the plastification of polymer in the weld zone is insufficient due to the low temperatures. Furthermore the risk of damaging the surfaces of the part due to heating of the sonotrode contact area rises.

Effects on process deviations and surface defects on differential pictures are shown in figure 6.
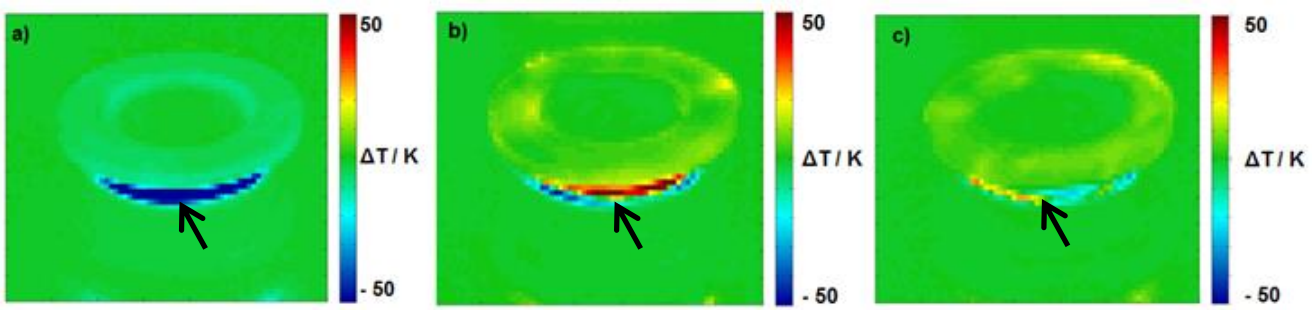

Fig. 6. Differential pictures of ultrasonic welded PA6 GF25 DVS specimens.

The weld path has been halved to $0.3 \mathrm{~mm}(\mathrm{a})$, the top part has been warmed prior to welding (b) or one half of the top part has been roughened (c). The weld area is indicated by an arrow.

A reduction of the weld path from $0.6 \mathrm{~mm}$ to $0.3 \mathrm{~mm}$ leads to an insufficient melting of the energy director accompanied by a lower energy input into the weld zone. This is why the weld area in the differential picture is around $50 \mathrm{~K}$ cooler than the reference weld. The weld quality is considerably lowered, as the improperly filled weld gap is acting as a notch in the weld. Additionally an overheating of the weld zone, e.g. by preheated weld parts, can also be detected by passive thermography as the weld zone gets hotter than in reference welds.

Surface defects like a roughened contact area (around $0.2 \mathrm{~mm}$ ) to the sonotrode are also visible in differential pictures making it possible to find parts with a poor surface quality. The roughened area leads to a higher energy conversion, visible through a temperature rise, on the roughened contact area. Correspondingly there is a lower energy input into the weld area leading to a cold area, thus a low surface quality may have an adverse effect on weld quality. Additionally there is a slight temperature increase beneath the unprepared area.

Table 4 is giving an overview over the detectability of several defects by passive thermography as well as mechanical test and visual inspection after ultrasonic welding.

Table 4. Detectability of different defects in ultrasonic welding by passive thermography, mechanical test (e.g. tensile test) and visual inspection after welding.

\begin{tabular}{|c|c|c|c|}
\hline \multirow{2}{*}{ Kind of defect } & \multicolumn{3}{|c|}{ Detectable by } \\
\hline & Passive thermography & Mechanical tests & Visual inspection \\
\hline \multicolumn{4}{|c|}{$\begin{array}{c}\text { Process deviations } \\
\end{array}$} \\
\hline $\begin{array}{l}\text { Weld path } \\
\text { (up to - } 50 \% \text { ) }\end{array}$ & Yes & Yes & No \\
\hline $\begin{array}{l}\text { Weld pressure } \\
\text { (up to + } 100 \mathrm{~N} \text { ) }\end{array}$ & Yes & Yes & No \\
\hline \multicolumn{4}{|c|}{ Part deviations } \\
\hline Surface roughness & Yes & No & Yes \\
\hline Surface defects & Yes & No & No \\
\hline $\begin{array}{l}\text { Polymer humidity } \\
\text { (up to } 3.84 \% \text { ) }\end{array}$ & Yes & Partly & Partly \\
\hline Filler aggregations & Yes & Yes & No \\
\hline
\end{tabular}

Process deviations as changes in weld path $(-50 \%)$ or weld pressure $(+100 \mathrm{~N})$ have not been detectable by visual inspection, but by passive thermography and mechanical tests. Furthermore, deviations like surface defects, e.g. notches in the energy director, or filler aggregations could not be seen by visual inspection, but are clearly visible in passive thermography. But for the mentioned defects only filler aggregations are relevant for the mechanical quality of the weld as they have been the only defect detectable in tensile tests. The detection of changes in polymer humidity by passive thermography has been possible for all considered humidities, whereas with mechanical test and visual inspection only a high humidity (3.84 \% in PA6 GF25) could be detected. At this water content the high attenuation of the ultrasonic waves leads to the melting of polymer on the parts surface. Thus passive thermography is highly sensitive to humidity changes in ultrasonic welding, as the attenuation of the ultrasound in the polymer changes severely with humidity. 


\section{Active thermography}

OLT and ULT have been used for the detection of common defects in hot plate welded and adhesive bonded polymer joints. The measurement times reached from a few minutes in ULT up to several hours in some cases during OLT.

\subsection{Optical lock-in thermography}

OLT for detection of air inclusions in overlapping adhesive bonds of different polymers are shown in figure 7.

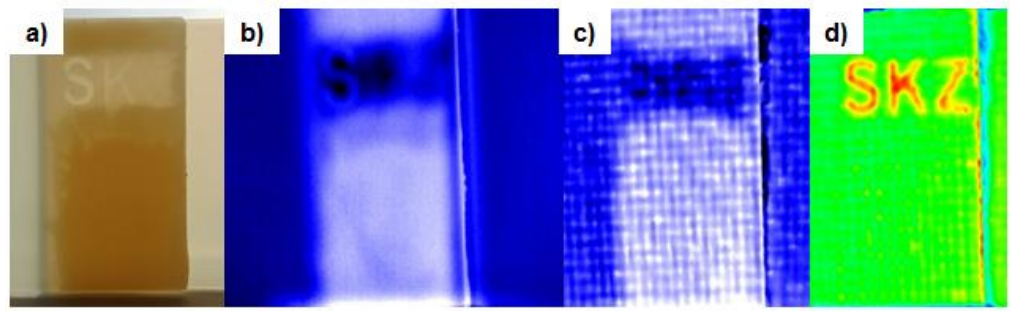

Fig. 7. Photograph of an overlapping adhesive bond of PA66 with an air inclusion building the letters 'SKZ' (a) and phase picture of the OLT (lock-in frequency $6.2 \mathrm{mHz}$ ) of this specimen (b).

The depth of the air inclusions is rising from ' $S$ ' $(1.0 \mathrm{~mm})$ to ' $\mathrm{Z}$ ' $(3.0 \mathrm{~mm})$.

Phase picture (c) and amplitude picture (d) of an equal specimen built from GFRP-PP (lock-in frequency $17.7 \mathrm{mHz}$ ).

It has been possible to detect air inclusions up to $1.0 \mathrm{~mm}$ depth (' $\mathrm{S}$ ') in PA66. Furthermore the missing application of adhesive around the area of the flaws, which can be seen in the photograph, is clearly visible in the phase pictures. In the phase picture of GFRP-PP the inclusion is slightly covered by additional reflections created by the fibreglass structures of the composite, slightly hindering the identification of the flaws shape. Apart from that the flaws are clearly visible up to a depth of $3.0 \mathrm{~mm}$ (' $Z$ ') in the amplitude picture, whereas the missing application of adhesive cannot be detected anymore. Thus it has to be kept in mind to use both, amplitude and phase picture, in order to properly detect flaws in polymer joints.

The detectability of several inclusions in hot plate welded PVC are shown in figure 8.
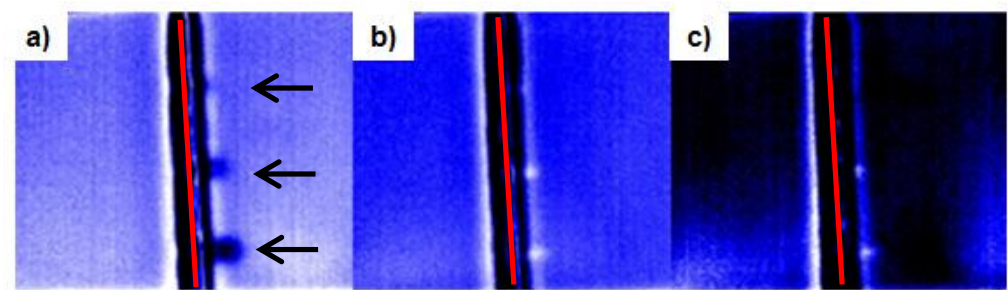

Fig. 8. Phase pictures (lock-in frequency $19.2 \mathrm{mHz}$ ) of hot plate welded PVC sheets.

The sheets have been prepared with drill holes $(2.5 \mathrm{~mm}$ diameter, $1.5 \mathrm{~mm}$ depth) to simulate air inclusions (a) and filled with metal (b) or sand (c) to simulate contaminations. The length of the drill holes increases from $5.0 \mathrm{~mm}$ (top) to $9.0 \mathrm{~mm}$ (bottom) in each picture. The weld area is indicated by a red line, the drill holes by arrows.

The air inclusions are visible in the phase pictures, but their shape is slightly blurred as the curvature of the drill holes leads to a diffuse reflection of the thermal waves. The detectability decreases for inclusions of metal and even more for inclusions of sand, as the coefficient of reflection for thermal waves are getting significantly smaller. So less of the thermal waves is being reflected towards the thermographic system. Generally defects have been detectable up to a depth of $2.5 \mathrm{~mm}$ with a minimum area of $5.0 \mathrm{~mm}^{2}$. For defect detection directly in the weld zone it is necessary to remove the weld bead, otherwise it will lead to shadowing effects as shown in figure 8. 


\subsection{Ultrasound lock-in thermography}

The detectability of inclusions in hot plate welded polymers has also been tested with ULT, an example of PA66 is shown in figure 9 .

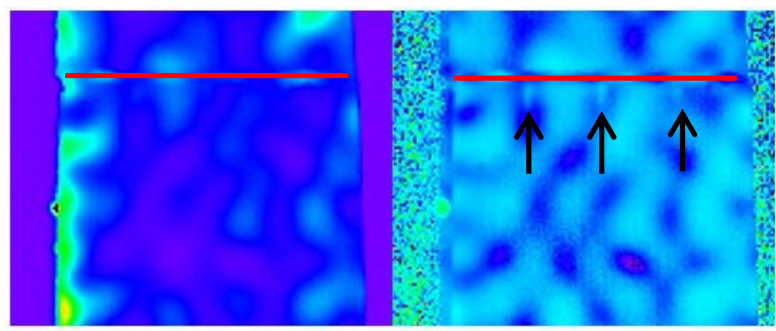

Fig. 9. Amplitude (left) and phase picture (right, lock-in frequency $20.0 \mathrm{mHz}$ ) of hot plate welded PA66 sheets. The sheets have been prepared with drill holes $(1.0 \mathrm{~mm}$ diameter, $1.5 \mathrm{~mm}$ depth) to simulate air inclusions. The length of the drill holes increases from $5.0 \mathrm{~mm}$ (left) to $9.0 \mathrm{~mm}$ (right) in each picture.

The weld area is indicated by a red line, the drill holes by arrows.

With ULT it has been possible to detect inclusions in comparable dimensions as with OLT, but with a better detectability of metallic inclusions, as the thermal waves are not reflected but directly generated at defect sites. Additionally the measurement times have usually been much smaller than with OLT. Nonetheless the detectability of sand is still poor in ULT. The achievable modulation of the ultrasound frequency in a band of $1.8 \mathrm{kHz}$ was not sufficient to suppress the generation of standing waves during excitation, which can be seen in the amplitude and phase pictures. These patterns slightly impede the detection of the defect sites, but even the small flaws shown in figure 9 are still detectable in the phase picture.

The detection of air inclusions in adhesive bonds of GFRP-PP is shown in figure 10.

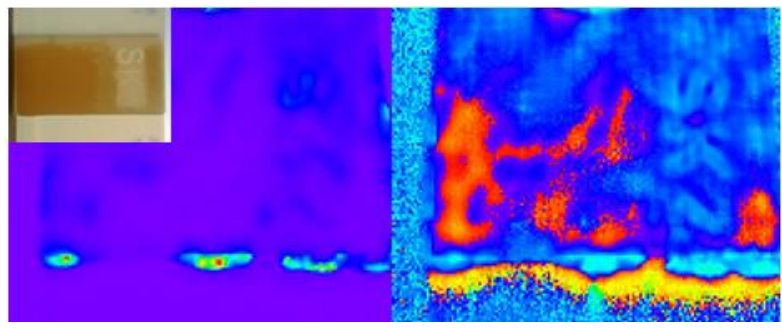

Fig. 10. Amplitude (left) and phase picture (right, lock-in frequency $40.0 \mathrm{mHz}$ )

of an overlapping adhesive bond of GFRP-PP with an air inclusion building the letters 'SKZ'.

The depth of the air inclusions is rising from ' $S$ ' $(1.0 \mathrm{~mm})$ to ' $\mathrm{Z}$ ' $(3.0 \mathrm{~mm})$.

The inset is showing the photograph of an equal specimen built from PA66.

With ULT it has been possible to detect the air inclusion ('SKZ') in the phase picture without interfering signals of the fibreglass structures which have been seen in OLT. The additional indications in the surrounding areas might occur due to a combination of standing wave patterns and an inhomogeneous application of adhesive. In this case, the amplitude picture did not contain any information on the hidden flaws. In contrast to that, the amplitude picture contains crucial information on the adhesive bond of GFRP-PA as shown in figure 11. 


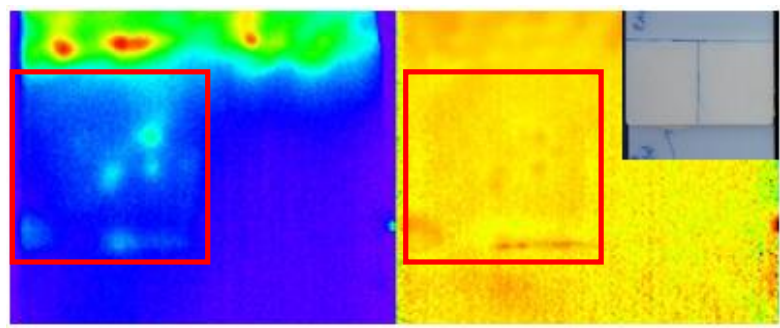

Fig. 11. Amplitude (left) and phase picture (right, lock-in frequency $40.0 \mathrm{mHz}$ )

of an overlapping adhesive bond of GFRP-PA with a contamination of oil on the left side of the bonding area (red square). The inset is showing the photograph of an equal specimen built from PA66.

One half of the joint has been wetted with a lubricant, leading to a weak adhesion between polymer and adhesive in this area. This area can be seen in the amplitude and phase pictures in ULT, as both sides of the joint may move against each other due to the weak adhesion and create heat through interfacial friction. The surrounding areas show no observable heat generation which gives rise to the high noise in these parts of the picture. The detectable spots in the lubricated area result from lubricant droplets inside the joint, which were created by coalescence.

\section{Conclusion}

In this work active and passive thermography have been shown as tools to assess defects in polymer joints. Passive thermography has been used to detect common defects and process deviations in welded polymer joints created by hot plate, infrared and ultrasonic welding. It was possible to detect deviations in the parts quality, e.g. wall thickness or surface roughness, as well as divergent process parameters, e.g. process temperatures or weld paths. In ultrasonic welding it has also been possible to detect differences in polymer humidity due to changed damping of the ultrasonic waves and a resulting heating of the polymer. Passive thermography gives fast feedback on the process and quality condition, because it is fast enough to be applicable as an on-line monitoring and easily automatable. But it has to kept in mind that not every nonconformity in passive thermography is a sign for a lowered product quality. Furthermore this method has some limitations, as the process has to generate heat which can be observed by the thermographic system in order to search for deviations.

Active thermography is not in the need of a process which inherently generates heat, as the heat is provided by external sources. Therefore active thermography can also be used for quality assurance apart from production processes, e.g. in use. With OLT and ULT it has been possible to detect inclusions of air, metal and sand up to a depth of $2.5 \mathrm{~mm}$ with a minimum area of $5.0 \mathrm{~mm}^{2}$ in hot plate welded polymer joints. Usually the measurement time to detect comparable defects has been much shorter for ULT (a few ten minutes) compared to OLT (minutes to hours), but is significantly longer than in passive thermography. Thus the use of these methods for on-line quality monitoring is limited to slow processes. As the weld bead leads to shadowing effects, it could be removed in order to locate defects directly in the weld zone.

In adhesive bonded polymer joints air inclusions in a depth of up to $3.0 \mathrm{~mm}$ had been detectable with both tested ways of excitation. Furthermore it has only been possible with ULT to detect areas with weak adhesion due to contaminations of the bonding surfaces with a lubricant. Also ULT did not produce additional signals due to the fibreglass structure of GFRPs. However the used ultrasound excitation system could not suppress the generation of standing waves, creating an additional pattern in the thermographic data. To fully suppress standing waves an ultrasound system with a broader bandwidth for frequency modulation has to be used.

\section{Acknowledgements}

The projects $17091 \mathrm{~N}$ and $18912 \mathrm{~N}$ of the research association FSKZ e.V. have been funded by the Arbeitsgemeinschaft industrielle Forschungsvereinigungen (AiF) as part of the programme Förderung der Industriellen Gemeinschaftsforschung (IGF) of the Bundesministerium für Wirtschaft und Energie (BMWi) based on a resolution of the German Bundestag. 


\section{REFERENCES}

[1] Kraus E., et al., Limits and possibilities in destructive testing of bonded polymer connections, Polymers Research Journal 9(3), pp. 337-344, 2015.

[2] Kraus E., et al., Fast detection of mechanical strength, Adhesion Adhesives\&Sealants 11(4), pp. 31-34, 2014.

[3] Hochrein T., et al., Nondestructive Test Methods: I Spy with my Little Eye Something that Is ..., Kunststoffe International 103, pp. 50-54, 2013.

[4] Horlemann S., et al., Passive thermography as a non-destructive testing procedure during the welding of plastics, Joining Plastics 9(2), pp. 2-9,2015.

[5] Riegert G., Induktions-Lockin-Thermografie: ein neues Verfahren zur zerstörungsfreien Prüfung, Dissertation, 2007. http://dx.doi.org/10.18419/opus-1734.

[6] Ibarra-Castenado C., et al., Comparative study of active thermography techniques for the nondestructive evaluation of honeycomb structures, Research in Nondestructive Evaluation 20(1), pp. 1-31, 2009.

[7] Oswald-Tranta, B., Time and frequency behavior in TSR and PPT evaluation for flash thermography, Quantitative InfraRed Thermography Journal 14(2), pp. 164-184, 2017.

[8] Gralewicz, G., Boguslaw W., Active thermography in qualitative evaluation of protective materials, International Journal of Occupational Safety and Ergonomics 15(4), pp. 363-371, 2015.

[9] Busse G., Optoacoustic phase angle measurement for probing a metal, Applied Physics Letters 35, pp. 759-760, 2007.

[10] Lehto A., et al., Amplitude and phase in thermal wave imaging, Electronics Letters 17, pp. 364-365, 1981.

[11] Uo P. K., et al., Parallel thermal wave imaging using a vector lock-in video technique, Photoacoustic and Photothermal Phenomena, Springer-Verlag, Heidelberg, pp. 415-418, 1987.

[12] Rosencwaig A., Busse G., High resolution photoacoustic thermal wave microscopy, Applied Physics Letters 36, pp. 725-727, 1980.

[13] Rosencwaig A., Photoacoustic microscopy, American Laboratory 11, pp. 39-49, 1979.

[14] Gleiter A., et al., Ultrasound-Lockin-Thermography for Advanced Depth Resolved Defect Selective Imaging, European Conference on NDT, Berlin, 2006.

[15] Wu D., et al., Inspection of aircraft structural components using lockin-thermography, Quantitative Infrared Thermography, pp. 251-256, 1996.

[16] Carlomagno G. M., Berardi, P. G., Unsteady thermotopography in non-destructive testing, Proc. $3^{\text {rd }}$ Biannual Exchange, pp. 33-39, St. Louis, 1976.

[17] Beaudoin J. L., et al., Numerical system for infrared scanners and application to the subsurface control of materials by photothermal radiometry, Infrared Technologies and Applications, SPIE 590, p. 287, 1985.

[18] Zweschper T., et al., Ultrasound excited thermography - advances due to frequency modulated elastic waves, Quantitative InfraRed Thermography Journal 2(1), pp. 65-76, 2005.

[19] Guo X., An analytical model and parametric analysis of ultrasound-excited infrared thermography, Quantitative InfraRed Thermography Journal 12(2), pp. 137-148, 2015.

[20] Gleiter A., Spießberger C., Busse G., Improved ultrasound activated thermography using frequency analysis, Quantitative InfraRed Thermography Journal 4(2), pp. 155-164, 2007.

[21] Stärk F., Temperature measurements on cyclically loaded materials, Werkstofftechnik 13, pp. 333-338, 1982.

[22] Mignogna R. B., et al., Thermographic investigations of high-power ultrasonic heating in materials, Ultrasonics 7, pp. 159-163, 1981.

[23] Rantala J, Wu D., Busse G., Amplitude Modulated Lock-In Vibrothermography for NDE of Polymers and Composites, Research in Nondestructive Evaluation 7, pp. 215-218, 1996.

[24] Zweschper Th., et al., Ultraschallangeregte Thermografie mittels frequenzmodulierter elastischer Wellen, DGZfP Thermografie-Kolloquium, Stuttgart, 2003.

[25] Dillenz A., Zweschper Th., Busse G., Progress in ultrasound phase thermography, SPIE 4360, pp. 576-579, Bellingham, 2001. 